\title{
Insights into eisosome assembly and organization
}

Kyoungtae Kim

Missouri State University

E. Murphy

MSU Graduate Student

Follow this and additional works at: https://bearworks.missouristate.edu/articles-cnas

\section{Recommended Citation}

Murphy, E. R., and K. T. Kim. "Insights into eisosome assembly and organization." Journal of biosciences 37, no. 2 (2012): 295-300.

This article or document was made available through BearWorks, the institutional repository of Missouri State University. The work contained in it may be protected by copyright and require permission of the copyright holder for reuse or redistribution.

For more information, please contact BearWorks@library.missouristate.edu. 


\title{
Review
}

\section{Insights into eisosome assembly and organization}

\author{
Murphy $\mathrm{ER}^{1}$ and KIM $\mathrm{KT}^{2, *}$ \\ ${ }^{1}$ Department of Molecular Genetics and Cell Biology, University of Chicago, Chicago, IL 60637, USA \\ ${ }^{2}$ Department of Biology, Missouri State University, Springfield, MO 65897, USA \\ *Corresponding author (Email, kkim@missouristate.edu)
}

\begin{abstract}
Eisosomes, large protein complexes that are predominantly composed of BAR-domain-containing proteins Pill and its homologs, are situated under the plasma membrane of ascomycetes. A successful targeting of Pill onto the future site of eisosome accompanies maturation of eisosome. During or after recruitment, Pill undergoes self-assembly into filaments that can serve as scaffolds to induce membrane furrows or invaginations. Although a consequence of the invagination is likely to redistribute particular proteins and lipids to a different location, the precise physiological role of membrane invagination and eisosome assembly awaits further investigation. The present review summarizes recent research findings within the field regarding the detailed structural and functional significance of Pill on eisosome organization.
\end{abstract}

[Murphy ER and Kim KT 2012 Insights into eisosome assembly and organization. J. Biosci. 37 295-300] DOI 10.1007/s12038-012-9206-6

\section{Introduction: Central organizers of eisosome}

The plasma membrane is a diverse structure that manages the traffic of materials in and out of the cell. It consists of dynamic compartments where different functions take place. These compartments exist over a wide range of spatial and temporal scales (Lingwood and Simons 2010). The budding yeast plasma membrane has been subdivided into three distinct membrane compartments: the membrane compartment of Can1 (MCC), the membrane compartment of TORC2 (MCT) and the membrane compartment of Pmal (MCP) (Young et al. 2002; Malinska et al. 2003; Grossmann et al. 2007; Berchtold and Walther 2009; Brach et al. 2011). MCC and MCT are found as distinct domains, whereas the MCP is found throughout the membrane except where a MCC or a MCT exists (Grossmann et al. 2007; Berchtold and Walther 2009). The cytoplasmic side of MCC appears to be closely associated with the protein cluster containing thousands of copies of Pill and its homolog Lsp1 (Grossmann et al. 2007; Frohlich et al. 2009). The cytosolic cluster was so named 'eisosome' (meaning portal for body in Greek) by Walther and coworkers because of its implication in endocytosis (Walther et al. 2006). It has been reported that in total 22 proteins ( 9 transmembrane and 13 cytoplasmic proteins) are members of MCC-associated proteins (Grossmann et al.
2008; Deng et al. 2009). Since the fluorescence microscope does not provide sufficient spatial resolution to differentiate between MCC and eisosome, Stradalova et al. (2009), using electron microscopy approaches, showed the ultrastructure of MCC that structurally resembles a furrow-like endocytic invagination. They showed that the transmembrane MCC marker Sur7 was found to localize to the superficial parts of the invaginated plasma membrane, while the cytosolic eisosome marker Pill was detected in the deeper parts of the furrow-like invagination mainly around the curved bottom of the structure (Stradalova et al. 2009). Upon deletion of budding yeast PIL1, GFP-fused eisosome (Lsp1, Slm1, Pkh1 and Pkh2) and MCC (Sur7, Nce102 and Can1) markers are localized to a few bright peripheral clusters called eisosome remnants, instead of localizing to the cell cortex evenly in a punctuated pattern (Walther et al. 2006, 2007; Frohlich et al. 2009; Grossmann et al. 2008; Kamble et al. 2011), indicating Pill is essential for the structural integrity of eisosome and MCC. Although Pill exhibits high levels of sequence homology among fungi, it seems that the function of Pill has not been completely conserved between budding yeast and other fungal species (Vangelatos et al. 2010; Kabeche et al. 2011; Reijnst et al. 2011; Seger et al. 2011). For instance, unlike budding yeast Pill (ScPil1) implicated in endocytosis (Walther et al. 2006; Murphy et al.

Keywords. Eisosome; membrane organization; Pill 
2011), Ashbya gossypii Pill (AgPil1) and Candida albicans Pill ( $\mathrm{CaPil1}$ ) are required for polar growth and essential for cell growth, respectively (Reijnst et al. 2011; Seger et al. 2011). The fact that the fission yeast orthologs of SIm1 and Sur7 do not colocalize with the MCC/eisosome marker Pill or depend on Pill for their localization in S. pombe also suggests the functional divergence of Pill in organizing $\mathrm{MCC} /$ eisosome between budding yeast and fission yeast (Kabeche et al. 2011).

\section{Up-to-date model for Pil1 assembly onto eisosome}

Recent studies discovered that both Pill and Lsp1 contain a BAR domain, structurally most similar to N-BAR domain which is also found in amphiphysins (Olivera-Couto et al. 2011; Ziolkowska et al. 2011). BAR domains consist of a coiled-coil of three long alpha-helices that dimerize to form a banana-shaped domain with a positively charged concave surface. Cationic residues on the positive surface interact with anionic membrane lipids via electrostatic interactions (Frost et al. 2009). Owing to the rigid concave shape of the surface, the BAR domain can induce membrane curvature, required for a wide range of cellular processes such as endocytic invagination and cell motility (Gallop and McMahon 2005; Suetsugu et al. 2010). The BAR-domaincontaining eisosome proteins Pill and Lsp1 are capable of self-assembly, binding lipid membranes, preferably those containing $\mathrm{PI}(4,5) \mathrm{P}_{2}$ (or $\mathrm{PIP}_{2}$ ), and deforming them into long tubules (Kabeche et al. 2011; Karotki et al. 2011; OliveraCouto et al. 2011). As expected, the positive surface patch of theoretical Pill homology modelling on the structural template of Lsp1 turned out to be important for its membrane binding and normal eisosome organization (Karotki et al. 2011; Olivera-Couto et al. 2011; Ziolkowska et al. 2011). Karotki et al. (2011) further proposed that, in addition to the positive residues on the concave surface, an N-terminal segment of Pill or Lsp1 might facilitate their efficient membrane binding and/or bending.

\section{Other protein factors implicated in organizing eisosome}

Beside the essential eisosomal organizing factor Pill, according to a genome-wide screen for deletion mutants that show an alteration of Pill localization, a wide range of genes (a total of 88 genes), functioning ranging from endocytosis and vesicle trafficking to metabolism, appear to be involved in the organization of the eisosome (Frohlich et al. 2009). Additionally, a similar genome-wide screen for deletion mutants that shows an altered Can1 (MCC marker) pattern identified 27 genes (Grossmann et al. 2008). Given eisosome and MCC are tightly associated with each other, it is striking to note that there was such a low level of consistency between those screens that in both screens NCE102, SUR4 and $M N N 10$ genes were the only overlapping ones that affect both MCC and eisosome organizations (Grossmann et al. 2008; Frohlich et al. 2009). One straightforward explanation for this would be that the recruitment of MCC and eisosome markers is differently regulated by non-overlapping factors in general, perhaps pointing out a subtle functional difference between MCC and eisosome. Except for Mnn10, the two other gene products, Sur4 and Nce102, are likely to influence eisosome organization through sphingolipid signalling by altering the level of sphingolipids and serving as a sphingolipid sensor in the plasma membrane, respectively (Han et al. 2002; Paul et al. 2006; Frohlich et al. 2009). Interestingly, it was found that the recruitment of Nce102, a bona fide MCC transmembrane protein, depends on the availability of sphingolipids at MCC where Nce102 acts as a negative regulator of Pkh kinases in Pill phosphorylation (Frohlich et al. 2009). Although more than 100 genes/proteins, to date, have been presented by the genetic screens to be important for $\mathrm{MCC} /$ eisosome organization, many more await further identification. This is because all essential genes ( $18 \%$ of the yeast genome) and a considerable number of proteins that show functional redundancy with other proteins in the yeast genome were excluded from the lists. For the latter case, $\operatorname{Slm} 1$ and its homolog $\operatorname{Slm} 2$ share the same function in organizing the eisosome as shown by Kamble et al. (2011), but Slm2 is not included on either list of genome-wide screens. Slm $1 / 2$ were originally characterized as $\mathrm{PIP}_{2}$ binding proteins through their C-terminal $\mathrm{PH}$ domain (Audhya et al. 2004; Fadri et al. 2005) and later found to be eisosome components (Grossmann et al. 2008; Kamble et al. 2011). A first clue to the targeting of Slm proteins to eisosome was provided by a recent microscopic study that clearly showed the central Slm (showing some sequence similarity to F-BAR) and PH domains are essentially required for eisosome targeting, but not by $\mathrm{PH}$ or F-BAR alone (Olivera-Couto et al. 2011), and thus underlining the significance of BAR domain on eisosome targeting.

\section{Reversible phosphorylation and its consequences on eisosome organization}

Another set of redundant genes that are not included in the genome list but important for eisosome organization are PKH1 and PKH2 (Walther et al. 2007), encoding the two mammalian PDK1 homologs Pkh1 and Pkh2 (Casamayor et al. 1999). The serine/threonine kinases Pkh1/2 are physically associated with the eisosome, and Pil1 and Lsp1 have been shown to be Pkh substrates in vitro (Zhang et al. 2004; Walther et al. 2007). Pill phosphorylation in vivo has been proposed to be an important regulator that affects eisosome assembly, since change in the phosphorylation level of Pill 
leads to defects in eisosome organization (Walther et al. 2007; Luo et al. 2008). However, controversy lies in the precise role of Pkh1/2-mediated phosphorylation of Pill on eisosome assembly. Walther et al. (2007) originally showed that hyperphosphorylation of Pill by elevated Pkh1 and Pkh2 protein levels leads to a severe defect in Pill assembly on eisosome (Walther et al. 2007). In support, they observed that the phospho-mimicking pill(4D) mutant in which serines 45, 59 and 230, and threonine 233 were changed to Aspartic acid (D) was dispersed mainly into the cytoplasm, thereby concluding that Pill is dephosphorylated when eisosome-bound and released upon phosphorylation to the cytoplasm (Walther et al. 2007) (figure 1A). The purified recombinant pill4(D) protein indeed was less competent in self-assembly compared to wild-type Pill, pointing to the role of Pill phosphorylation that leads to impairment of Pill assembly on eisosome (Karotki et al. 2011). In agreement, Deng et al. (2009) reported that the decreased Pill-GFP fluorescence level in the cytoplasm correlates with the dephosphorylation of Pill, especially at the sites of Ser-230

\section{(A) (Walther et al. 2007)}

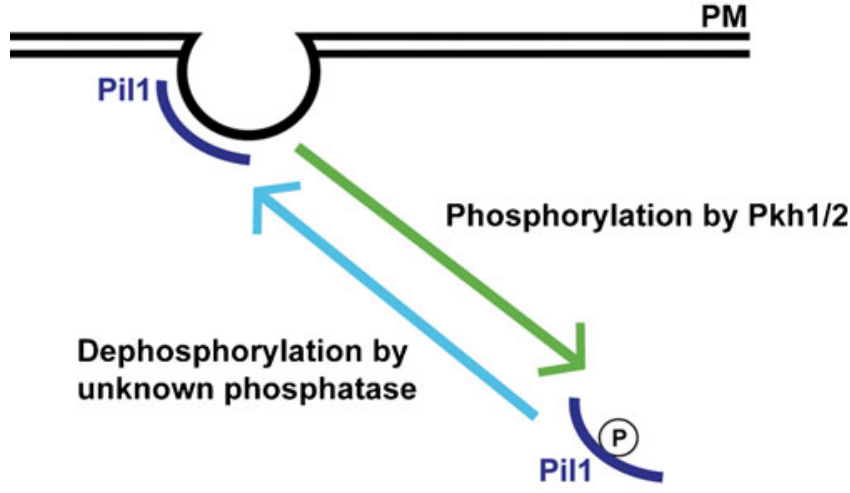

(B) (Lou et al. 2008)

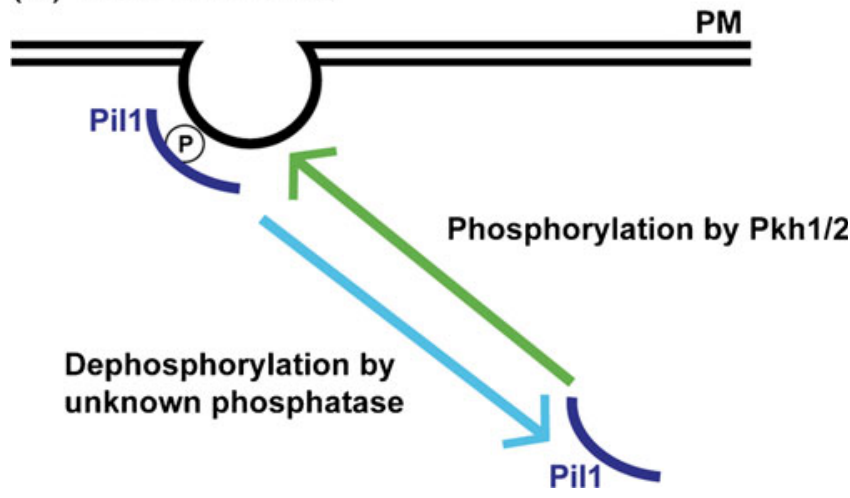

Figure 1. The controversy of the roles of reversible phosphorylation of Pill in eisosome organization. (A) The model suggests that Pill dephosphorylation promotes its recruitment to the eisosome. (B) The other model proposes that dephosphorylation of Pill is required for its disassembly from the eisosome. and Thr-233. However, two lines of evidence argue against the notion that the dephophorylated Pill associates with eisosome. First, according to Luo et al. (2008), a nonphosphorylable Pill mutant in which multiple phosphorylation sites (up to six) were mutated to Alanine was mislocalized to the cytoplasm, suggesting that eisosome formation requires phosphorylation of Pill (figure 1B). Consistent with this observation, the addition of KP-372-1, which inhibits Pkh1/2 kinases, caused the increase of the pool of dephosphorylated Pill, primarily situated in the cytoplasm, supporting the view that Pill phosphorylation is required for eisosome assembly (Baxter et al. 2011). At the moment it is not clear how different groups of researchers obtained two opposing results using similar Pill mutants. Nonetheless, the notion of reversible Pill phosphorylation and dephosphorylation controlling structural integrity of eisosome is highly acceptable. The existence of a phosphatase system involved in Pill dephosphorylation was suggested by Deng et al. (2009) as they observed an abrupt decrease in Pill and Lsp1 phosphorylation level during cell cycle. One potential candidate phosphatase for dephosphorylation of Pill would be calcineurin, which is known to be directly dephosphorylate another eisosome members $\operatorname{Slm} 1 / 2$ (Bultynck et al. 2006). If so, the consequence of Pill and Lsp1 dephosphorylation by calcineurin or other phosphatases yet to be identified on eisosome assembly should be further investigated to establish the biochemical recruitment mechanisms of Pill.

\section{Suggested roles of membrane lipids on eisosome}

The plasma membrane is made up of several types of lipids, and these lipids play an important role in the recruitment of proteins. Sphingolipids consist of long saturated acyl chains that allow them to pack together tightly in the membrane (Brown and London, 2000). The micro-domain of the plasma membrane enriched in sphingolipids and cholesterol (Lemaire-Ewing et al. 2011) is often called a lipid raft, which is essential in cell signalling and protein trafficking (Staubach and Hanisch 2011). An early detergent lipid raft extraction assay showed that Can1, an integral MCC protein, localizes in lipid rafts and that a reduction in sphingolipids leads to the disruption of Can1 targeting (Malinska et al. 2003). The recruitment of another integral MCC protein Nce102 was also found to be dependent on a higher level of sphingolipids (Frohlich et al. 2009). Likewise, decreased content of sphingolipids in lcb1-100 mutant (Zanolari et al. 2000) or treatment of myriocin that inhibits sphingolipid synthesis resulted in a loss of the MCC-associating cytoplasmic eisosome carrying Pill (Walther et al. 2007; Luo et al. 2008; Frohlich et al. 2009). These results apparently support the notion that membrane lipids are required for correct intracellular targeting of raft-associated proteins (Hearn et al. 2003). $\mathrm{PI}(4,5) \mathrm{P}_{2}\left(\mathrm{PIP}_{2}\right)$ is a minor yet dynamic phospholipid 
component of the plasma membrane which assists in the recruitment of a wide range of proteins to the plasma membrane (Strahl and Thorner 2007). Indeed, this was the case for Can 1 targeting to the periphery of the plasma membrane; in $m s s 4^{t s}$ temperature sensitive mutant that has $\sim 10 \%$ of normal amount of $\mathrm{PIP}_{2}$ (Desrivieres et al. 1998), the targeting of MCC protein Can1 was found to be impaired (Daquinag et al. 2007). However, the phenotypic defect in Can1 targeting in the mutant appears to be a secondary effect, perhaps caused by an actin cytoskeleton defect. More recently, Karotki et al. (2011) established a direct role of $\mathrm{PIP}_{2}$ in organizing eisosome in vivo. In lower levels of $\mathrm{PIP}_{2}$ (in $m s s 4^{t s}$ ), Pill progressively dissociated from the plasma membrane, mainly due to the fact that the probability of its direct interaction with $\mathrm{PIP}_{2}$ significantly decreases while in an opposing condition (in sjll $\Delta$ sjl2 $\Delta$ ) with higher chance, thus forming enlarged Pill puncta (Karotki et al. 2011) (E Murphy, unpublished). The fungal-specific sterol, ergosterol, enriched in lipid rafts, is another major lipid that plays a role in aiding the targeting of lipid-raft associating protein factors such as Gas1 and Pmal (Bagnat et al. 2000). At least notable, but not complete, mistargeting of the MCC marker Can1 was observed in the condition where ergosterol levels were reduced (erg6s and $\operatorname{erg} 24 \Delta$ ) (Malinska et al. 2003). In contrast, unpublished data from Walther laboratory (Frohlich et al. 2009), including ours, strongly argue for a negligible role of ergosterol in targeting of the eisosome protein Pill, since GFP fused Pill in those sterol mutant strains was still properly targeted to the plasma membrane.

\section{Physiological functions of eisosome}

It is curious that the liquid phase endocytic marker FM4-64 shows partial colocalization with Pill (Walther et al. 2006) (E Murphy, unpublished), and that the extent of spatial overlap between the eisosome and FM4-64 appears to increase as aberrant eisosome aggregates are formed by the loss of Pill. However, it is now generally accepted that the eisosome does not mark receptor-mediated endocytic (RME) sites, on the basis of several lines of evidence: (1) the plasma membrane MCC protein Sur7, which colocalizes to the eisosome (Malinska et al. 2004; Walther et al. 2006), does not colocalize with RME sites carrying Rvs161 and Ede1 (Grossmann et al. 2008); (2) none of Abp1- and Sla1-GFP endocytic sites colocalized with Pill-mCherry (Brach et al. 2011) and (3) Slm1, an eisosome marker, displayed only rare colocalization with Abp1-GFP, raising the possibility of random colocalization between them (Kamble et al. 2011). Nevertheless, what has emerged clearly is that a stable eisosome structure at the cell cortex is required for efficient receptor-mediated endocytosis occurring in the vicinity of the eisosome. This notion is supported by the observation that the rate of Ste3-mediated (a factor receptor) endocytosis to the vacuole in pill $\Delta$ and $l s p l \Delta$ cells decreased significantly when compared to that of WT cells (Walther et al. 2006). Notably, the efficacy of receptor-mediated endocytosis dropped significantly in PIL1-lacking cells in which the synaptojanins (Sj11/2) were severely mislocalized to the cytoplasm (Murphy et al. 2011). In particular, $\mathrm{Sj} 12$ is a major plasma membrane phosphoinositide phosphatase that hydrolyses phosphates of $\mathrm{PIP}_{2}$ (Guo et al. 1999), and a transient reduction of $\mathrm{PIP}_{2}$ or the change of $\mathrm{PIP}_{2}$ levels in a temporal manner via $\mathrm{Sj} 12$ at endocytic sites is known to be critical for the efficiency of endocytosis (Sun et al. 2007; Toret et al. 2008). Thus, the failure of Sj12 targeting to endocytic sites, most likely a side effect caused by loss of Pill (Murphy et al. 2011), might lead to a cascading failures in which an unsuccessful spatiotemporal regulation of $\mathrm{PIP}_{2}$ levels, triggers the failure of endocytosis. At the moment it is plausible to propose that Pill is directly or indirectly involved in fine-tuning to regulate membrane phospholipid homeostasis. Furthermore, it appears that MCC/eisosome is a protective area that provides stability for the proteins localized there; the MCC component Can1 (transmembrane arginine transporter) was dissipated throughout the cell membrane and endocytosed at a much faster rate in pill $\Delta$ cells than it is in WT cells, most likely due to the lack of the protective barrier in the mutant strain (Grossmann et al. 2008). It is yet important to note that the protective role of eisosome for its protein component from endocytosis seems not to be unanimously supported, based on the finding that the endocytosis rates of Can1 in pill $\Delta$ and WT cells were essentially the same (Brach et al. 2011).

\section{Concluding remarks}

Work over the last 5-6 years has been focused on the contributions of Pill and its homologs in ascomycetes to $\mathrm{MCC} /$ eisosome organization. As discussed, a new, and we believe potentially very important piece of information regarding the structure and function of Pill, has come with the recent finding that Pill, containing a BAR domain, is able to selfassemble into filaments that serve as scaffold to reorganize membrane into an invagination. While evidence is mounting for a dynamic Pill assembly required for eisosome/MCC organization, precisely how these Pill fibres are used to help provide the force required for the invagination is not understood fully. In light of finding that inactivation of the PKC kinases Pkh1 and Pkh2 leads to the formation of extended net-like eisosome carrying Pill, one can postulate that there must be a primary signal pathway with $\mathrm{Pkh} 1 / 2$ kinases that serve as a negative regulator of Pill assembly, as well as certain factors, including Nce102 that plays opposing regulatory roles. Therefore, in the future it is of great interest to understand the new and detail functions of already-known and yet-to-be-identified factors that influence Pill assembly 
in vitro, and to further elucidate the physiological relevance of the factors in eisosome assembly in vivo. Along with at least learning more details about Pill-lipids interaction, it is highly likely in the next few years to gain a better understanding of how the interaction of Pill with transmembrane MCC and cytosolic eisosome proteins regulates MCC/eisosome organization. All together, these studies during the coming years should provide even greater insights to the understanding of biological membrane organization and function.

\section{References}

Audhya A, Loewith R, Parsons AB, Gao L, Tabuchi M, Zhou H, Boone C, Hall MN, and Emr SD 2004 Genome-wide lethality screen identifies new PI4,5P2 effectors that regulate the actin cytoskeleton. EMBO J. 23 3747-3757

Bagnat M, Keranen S, Shevchenko A and Simons K. 2000 Lipid rafts function in biosynthetic delivery of proteins to the cell surface in yeast. Proc. Natl. Acad. Sci. USA 97 3254-3259

Baxter BK, Didone L, Oga D, Schor S and Krysan DJ 2011 Identification, in vitro activity and mode of action of phosphoinositide-dependent-1 kinase inhibitors as antifungal molecules. ACS Chem. Biol. 20 502-510

Berchtold D and Walther TC 2009 TORC2 plasma membrane localization is essential for cell viability and restricted to a distinct domain. Mol. Biol. Cell 20 1565-1575

Brach T, Specht T and Kaksonen M 2011 Reassessment of the role of plasma membrane domains in the regulation of vesicular traffic in yeast. J. Cell Sci. 124 328-337

Brown DA and London E 2000 Structure and function of sphingolipid- and cholesterol-rich membrane rafts. J. Biol. Chem. 275 17221-17224

Bultynck G, Heath VL, Majeed AP, Galan JM, Haguenauer-Tsapis $\mathrm{R}$ and Cyert MS $2006 \mathrm{Slm} 1$ and $\operatorname{sim} 2$ are novel substrates of the calcineurin phosphatase required for heat stress-induced endocytosis of the yeast uracil permease. Mol. Cell Biol. 264729 4745

Casamayor A, Torrance PD, Kobayashi T, Thorner J and Alessi DR 1999 Functional counterparts of mammalian protein kinases PDK1 and SGK in budding yeast. Curr. Biol. 9186 197

Daquinag A, Fadri M, Jung SY, Qin J and Kunz J 2007 The yeast PH domain proteins Slm1 and Slm2 are targets of sphingolipid signaling during the response to heat stress. Mol. Cell Biol. 27 633-650

Deng C, Xiong X and Krutchinsky AN 2009 Unifying fluorescence microscopy and mass spectrometry for studying protein complexes in cells. Mol. Cell. Proteom. 8 1413-1423

Desrivieres S, Cooke FT, Parker PJ and Hall MN 1998 MSS4, a phosphatidylinositol-4-phosphate 5-kinase required for organization of the actin cytoskeleton in Saccharomyces cerevisiae. J. Biol. Chem. 273 15787-15793

Fadri M, Daquinag A, Wang S, Xue T and Kunz J 2005 The pleckstrin homology domain proteins $\mathrm{Slm} 1$ and $\mathrm{Slm} 2$ are required for actin cytoskeleton organization in yeast and bind phosphatidylinositol-4,5-bisphosphate and TORC2. Mol. Biol. Cell 16 1883-1900

Frohlich F, Moreira K, Aguilar PS, Hubner NC, Mann M, Walter P and Walther TC 2009 A genome-wide screen for genes affecting eisosomes reveals Nce102 function in sphingolipid signaling. J. Cell Biol. 185 1227-1242

Frost A, Unger VM and De Camilli P 2009 The BAR domain superfamily: membrane-molding macromolecules. Cell 137 191-196

Gallop JL and McMahon HT 2005 BAR domains and membrane curvature: bringing your curves to the BAR. Biochem. Soc. Symp. 223-231

Grossmann G, Malinsky J, Stahlschmidt W, Loibl M, Weig-Meckl I, Frommer WB, Opekarova M and Tanner W. 2008 Plasma membrane microdomains regulate turnover of transport proteins in yeast. J. Cell Biol. 183 1075-1088

Grossmann G, Opekarova M, Malinsky J, Weig-Meckl I and Tanner W. 2007 Membrane potential governs lateral segregation of plasma membrane proteins and lipids in yeast. EMBO J. 26 $1-8$

Guo S, Stolz LE, Lemrow SM and York JD 1999 SAC1-like domains of yeast SAC1, INP52 and INP53 and of human synaptojanin encode polyphosphoinositide phosphatases. J. Biol. Chem. 274 12990-12995

Han G, Gable K, Kohlwein SD, Beaudoin F, Napier JA and Dunn TM 2002 The Saccharomyces cerevisiae YBR159w gene encodes the 3-ketoreductase of the microsomal fatty acid elongase. J. Biol. Chem. 277 35440-35449

Hearn JD, Lester RL and Dickson RC 2003 The uracil transporter Fur4p associates with lipid rafts. J. Biol. Chem. 278 3679-3686

Kabeche R, Baldissard S, Hammond J, Howard L and Moseley JB 2011 The filament-forming protein Pill assembles linear eisosomes in fission yeast. Mol. Biol. Cell 22 4059-4067

Kamble C, Jain S, Murphy E and Kim K 2011 Requirements of SIm proteins for proper eisosome organization, endocytic trafficking and recycling in the yeast Saccharomyces cerevisiae. J. Biosci. 36 79-96

Karotki L, Huiskonen JT, Stefan CJ, Ziolkowska NE, Roth R, Surma MA, Krogan NJ, Emr SD, Heuser J, Grunewald K, et al. 2011 Eisosome proteins assemble into a membrane scaffold. J. Cell Biol. 195 889-902

Lemaire-Ewing S, Lagrost L and Neel D 2011 Lipid rafts: A signalling platform linking lipoprotein metabolism to atherogenesis. Atherosclerosis 221 303-310

Lingwood D and Simons K. 2010 Lipid rafts as a membraneorganizing principle. Science 327 46-50

Luo G, Gruhler A, Liu Y, Jensen ON and Dickson RC 2008 The sphingolipid long-chain base-Pkh1/2-Ypk1/2 signaling pathway regulates eisosome assembly and turnover. J. Biol. Chem. 283 10433-10444

Malinska K, Malinsky J, Opekarova M and Tanner W 2003 Visualization of protein compartmentation within the plasma membrane of living yeast cells. Mol. Biol. Cell 14 4427-4436

Malinska K, Malinsky J, Opekarova M and Tanner W 2004 Distribution of Can $1 p$ into stable domains reflects lateral protein segregation within the plasma membrane of living $S$. cerevisiae cells. J. Cell Sci. 117 6031-6041 
Murphy ER, Boxberger J, Colvin R, Lee SJ, Zahn G, Loor F and Kim K 2011 Pill, an eisosome organizer, plays an important role in the recruitment of synaptojanins and amphiphysins to facilitate receptor-mediated endocytosis in yeast. Eur. J. Cell Biol. 90 825-833

Olivera-Couto A, Grana M, Harispe L and Aguilar PS 2011 The eisosome core is composed of BAR domain proteins. Mol. Biol. Cell 22 2360-2372

Paul S, Gable K, Beaudoin F, Cahoon E, Jaworski J, Napier JA and Dunn TM 2006 Members of the Arabidopsis FAE1-like 3-ketoacyl-CoA synthase gene family substitute for the Elop proteins of Saccharomyces cerevisiae. J. Biol. Chem. 281 9018-9029

Reijnst P, Walther A and Wendland J 2011 Dual-colour fluorescence microscopy using yEmCherry-/GFP-tagging of eisosome components Pill and Lsp1 in Candida albicans. Yeast 28 331-338

Seger S, Rischatsch R and Philippsen P 2011 Formation and stability of eisosomes in the filamentous fungus Ashbya gossypii. J. Cell Sci. 124 1629-1634

Staubach S and Hanisch FG 2011 Lipid rafts: signaling and sorting platforms of cells and their roles in cancer. Expert Rev. Proteom. $8263-277$

Stradalova V, Stahlschmidt W, Grossmann G, Blazikova M, Rachel R, Tanner W and Malinsky J 2009 Furrow-like invaginations of the yeast plasma membrane correspond to membrane compartment of Can1. J. Cell Sci. 122 2887-2894

Strahl T and Thorner J 2007 Synthesis and function of membrane phosphoinositides in budding yeast Saccharomyces cerevisiae. Biochim. Biophys. Acta 1771 353-404

Suetsugu S, Toyooka K and Senju Y. 2010 Subcellular membrane curvature mediated by the BAR domain superfamily proteins. Semin. Cell Dev. Biol. 21 340-349
Sun Y, Carroll S, Kaksonen M, Toshima JY and Drubin DG 2007 PtdIns(4,5)P2 turnover is required for multiple stages during clathrin- and actin-dependent endocytic internalization. J. Cell Biol. 177 355-367

Toret CP, Lee L, Sekiya-Kawasaki M and Drubin DG 2008 Multiple pathways regulate endocytic coat disassembly in Saccharomyces cerevisiae for optimal downstream trafficking. Traffic 9 848-859

Vangelatos I, Roumelioti K, Gournas C, Suarez T, Scazzocchio C and Sophianopoulou V. 2010 Eisosome organization in the filamentous ascomycete Aspergillus nidulans. Eukaryot. Cell 9 1441-1454

Walther TC, Aguilar PS, Frohlich F, Chu F, Moreira K, Burlingame $\mathrm{AL}$ and Walter P 2007 Pkh-kinases control eisosome assembly and organization. EMBO J. 26 4946-4955

Walther TC, Brickner JH, Aguilar PS, Bernales S, Pantoja C and Walter P 2006 Eisosomes mark static sites of endocytosis. Nature 439 998-1003

Young ME, Karpova TS, Brugger B, Moschenross DM, Wang GK, Schneiter R, Wieland FT and Cooper JA 2002 The Sur7p family defines novel cortical domains in Saccharomyces cerevisiae, affects sphingolipid metabolism and is involved in sporulation. Mol. Cell Biol. 22 927-934

Zanolari B, Friant S, Funato K, Sutterlin C, Stevenson BJ and Riezman H 2000 Sphingoid base synthesis requirement for endocytosis in Saccharomyces cerevisiae. EMBO J. 19 2824-2833

Zhang X, Lester RL and Dickson RC 2004 Pillp and Lsp1p negatively regulate the 3-phosphoinositide-dependent protein kinase-like kinase Pkh1p and downstream signaling pathways Pkc1p and Ypk1p. J. Biol. Chem. 279 22030-22038

Ziolkowska NE, Karotki L, Rehman M, Huiskonen JT and Walther TC 2011 Eisosome-driven plasma membrane organization is mediated by BAR domains. Nat. Struct. Mol. Biol. 18 854-856

MS received 19 January 2012; accepted 23 April 2012

Corresponding editor: LuIS M CORROCHANO 